قسم صحة الحيو ان ـ كلية الطب البيطرى جامعة البعث - حماه - سوريا.

\title{
A STUDY OF AIR CONTAMINATION BY BACTERIA AND FUNGI IN BROILER HOUSES
}

(With 7 Tables and 6 Figures)

\author{
By \\ M.M. ODABASHI and M. SALEH
}

(Received at 21/5/2011)

دراسة الملوثات الجرثومية والفظرية في هواء حظائر الفروج

ماهر موفق أوضه باشسي ، ماهرصالح

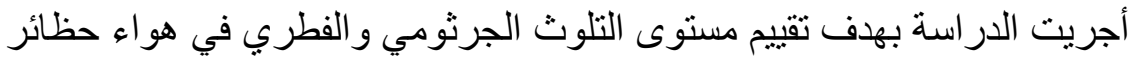

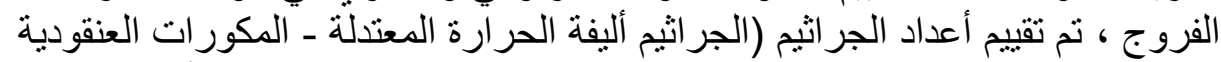

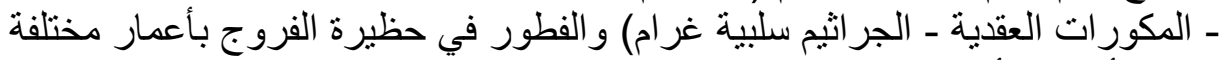

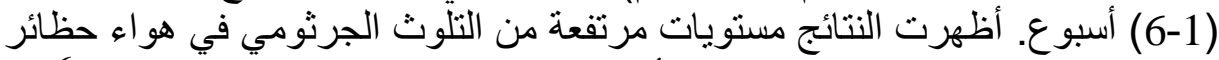

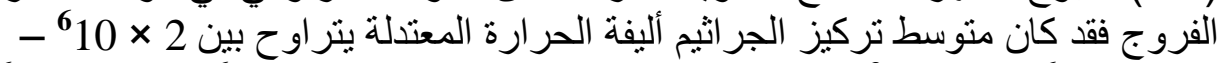

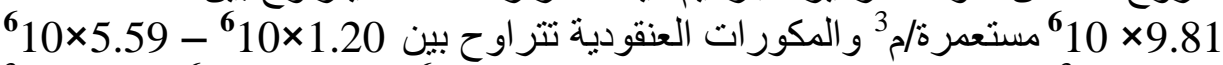

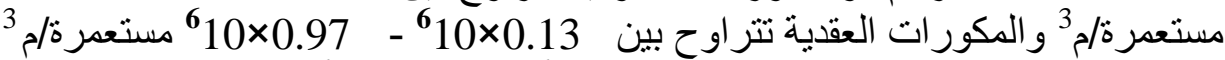

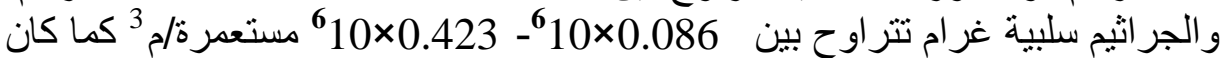

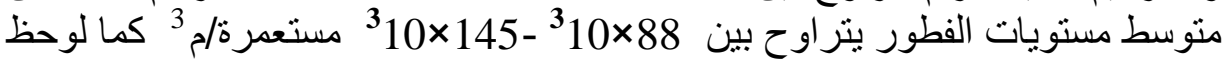

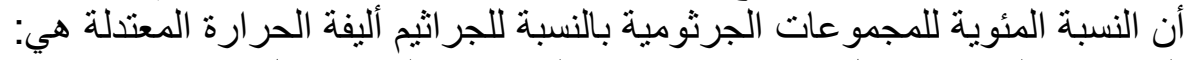

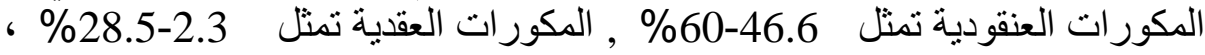

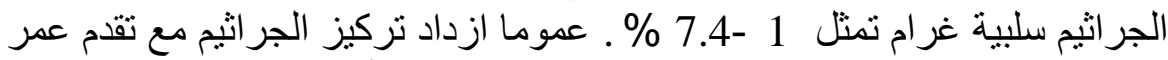

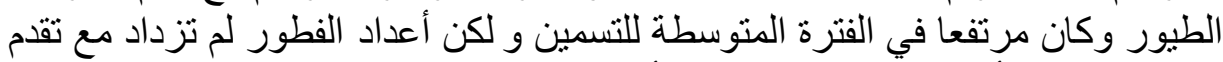

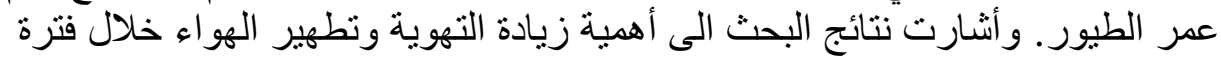

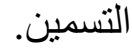

كلمات مفتاحية : الجراثيم أليفة الحرارة الدعتلة- الفطور- عدر الطيور- حظائر الفروج

\section{SUMMERY}


The aim of this paper was to assess the level of air contamination by bacteria and fungi in broiler houses. The numbers of bacteria (mesophile, staphylococci, streptococci and gram-negative) and fungi were determined in broiler houses with chicken of different ages (1-6 weeks old). The results showed high levels from bacterial contamination in broiler houses air. The mean number of bacteria ranged between $2 \times 10^{6}-9.81 \times 10^{6} \mathrm{cfu} / \mathrm{m}^{3}$ for the total number of mesophilic bacteria, between $1.20 \times 10^{6}-5.59 \times 10^{6} \mathrm{cfu} / \mathrm{m}^{3}$ for staphylococci, between $0.13 \times 10^{6}-0.97 \times 10^{6} \mathrm{cfu} / \mathrm{m}^{3}$ for streptococci, and between $0.086 \times 10^{6}-$ $0.423 \times 10^{6} \mathrm{cfu} / \mathrm{m}^{3}$ for gram-negatives. The mean fungi levels were middle and ranged between $88 \times 10^{3}$ to $145 \times 10^{3} \mathrm{cfu} / \mathrm{m}^{3}$. The proportions of bacterial groups within the total mesophilic bacteria number were: 46.6 - $60 \%$ staphylococci, 2.3 - $28.5 \%$ streptococci and $1-7.4 \%$ gramnegative. In general, the number of bactria increased with poultry age and it was high in the mid-fattening period but the number of fungi did not increase with the chicken's age. The results indicate the necessity for increased ventilation and for air disinfection during the chicken's fattening period.

Key words: Mesophilic bacteria, fungi, poultry age, broiler houses.

\section{INTRODUCTION المقدمـة}

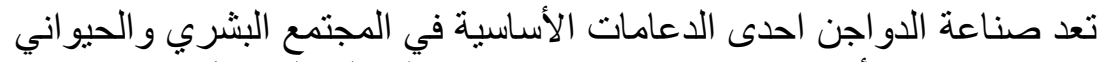

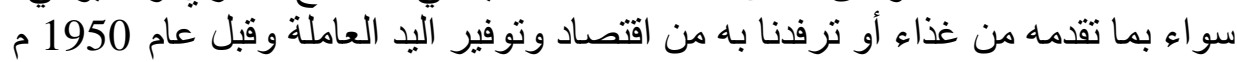

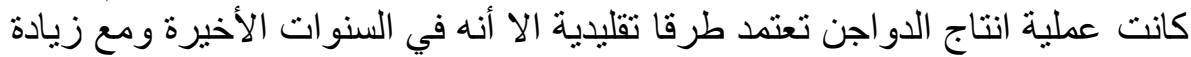

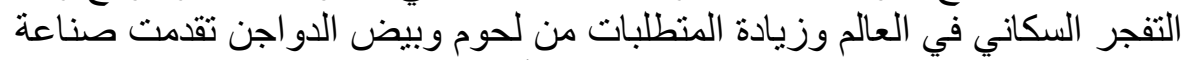

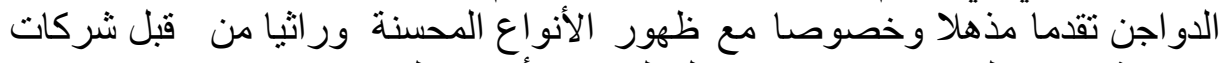

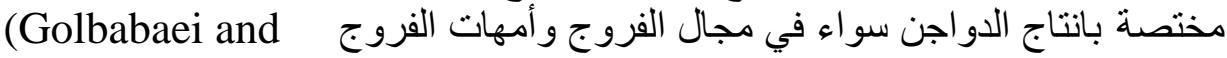
.Islami, 2000)

كما باتت الدر اسات العلمية والاقتصادية تحاول زيادة الربح عبر تقليل تكلفة

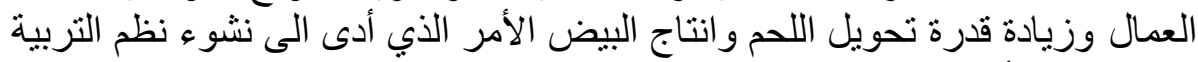

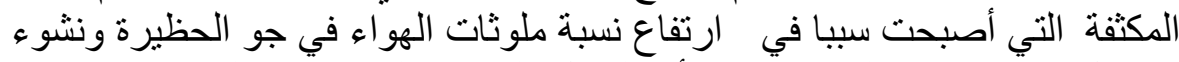

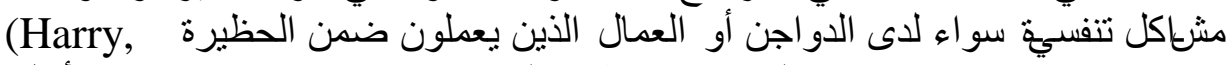

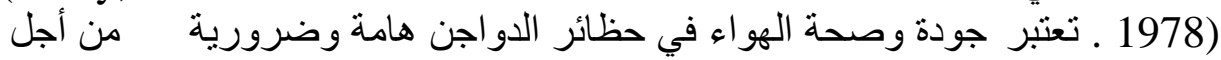

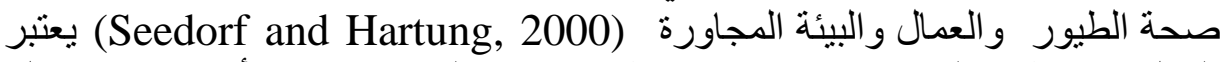
العمل في حظائر الدواجن مهنة ذات مخاطر تتفسية عالية في دراسة الجة أجريت في شمال 


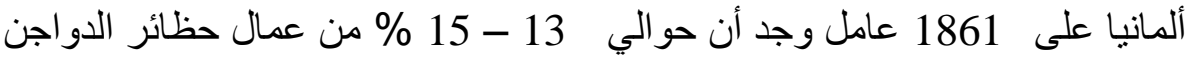

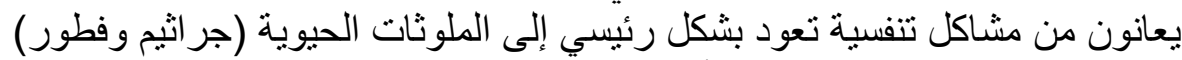

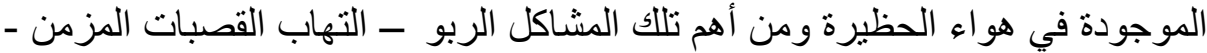

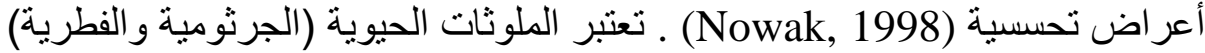

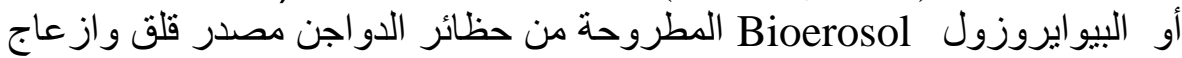

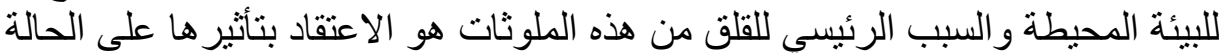

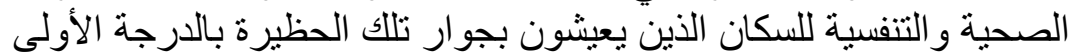
(Silvana et al., 2010) في الدرجـة الثانيـة (Muller and Wieser, 1987; Schulz et al., 2005).

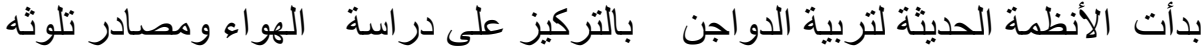

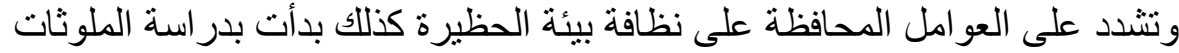

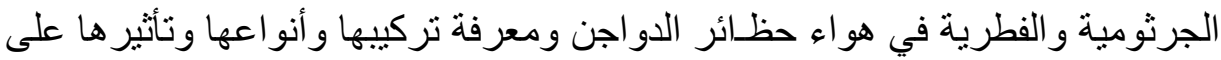

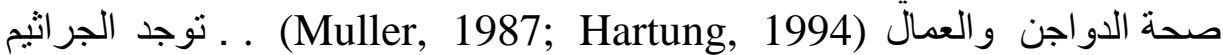

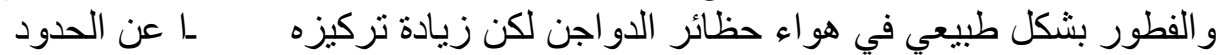

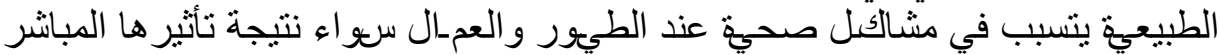

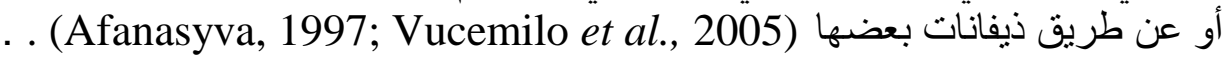

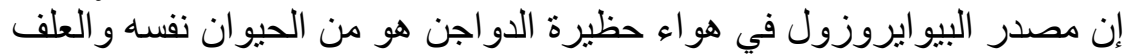

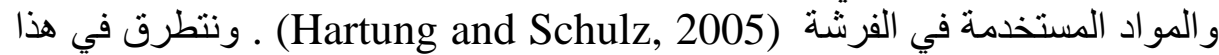

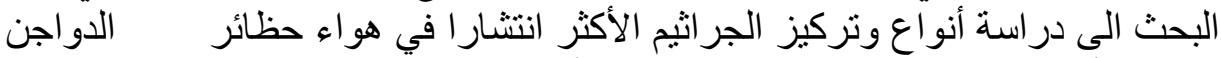

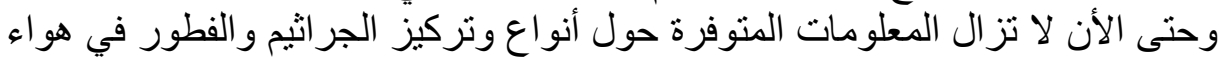

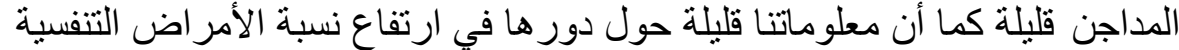

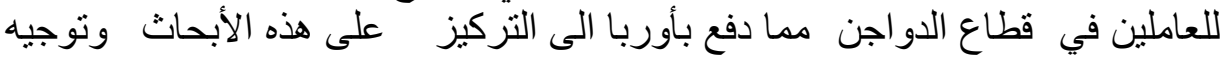

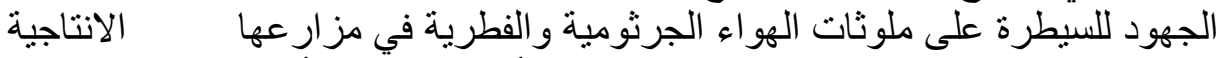

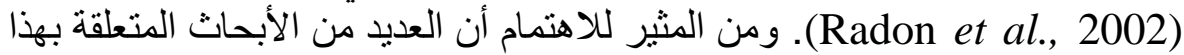

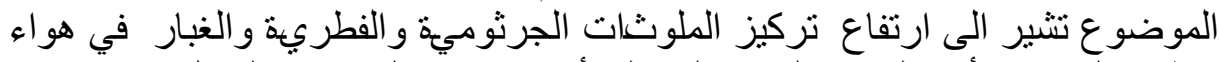

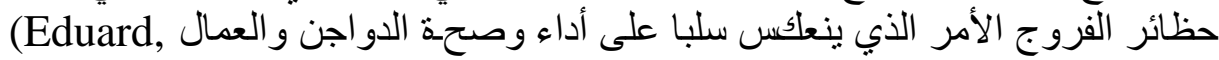
1997; Bakutis et al., 2004; Seedorf, 2005)

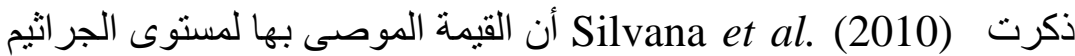

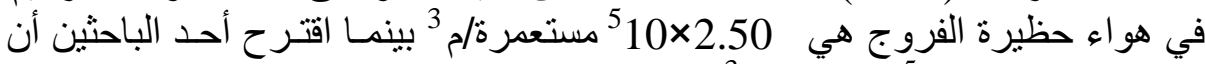

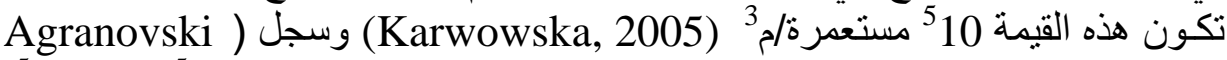

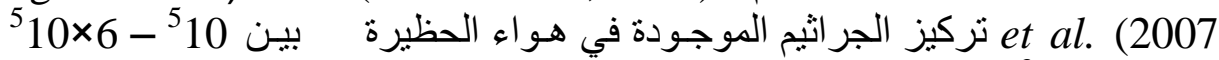

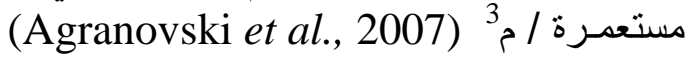

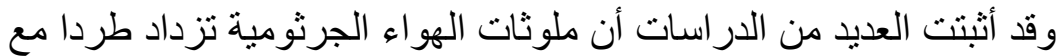

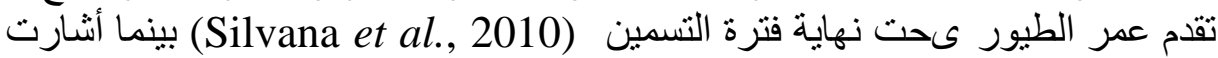

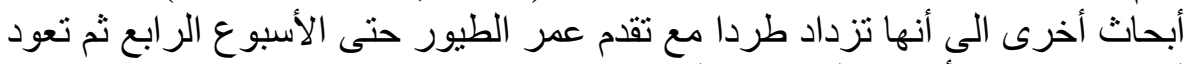

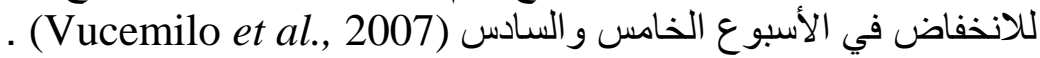


و أغلب المر اجع أشارت الى أن الأغلبية العظمى من الجر اثيم الموجودة في

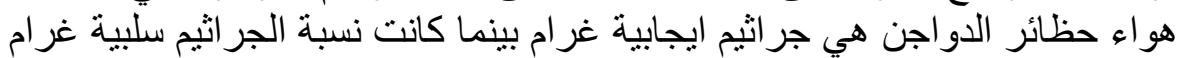

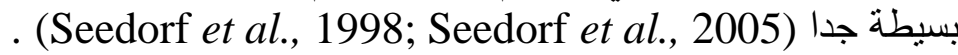
عزى الباحث (2005) Karwowska التلوث الهو ائي المرتفع في الحظيرة الى عدم كفاءة أنظمة التهوية المستخدمة والأعمال المنفذة ضمن الحظيرة .Karwowska (2005)

\section{MATERIALS and METHODS مواد وطرق البحث}

تتميز بينائها القديم وهي تتكون من من من من تم أخذ العينات من مزر عة للفروج

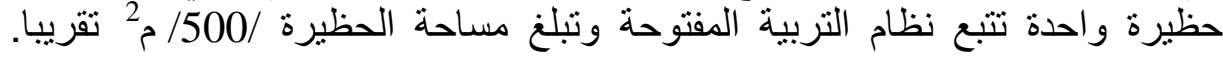

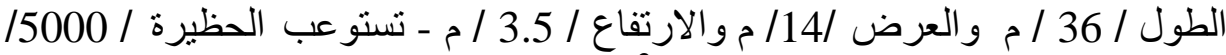

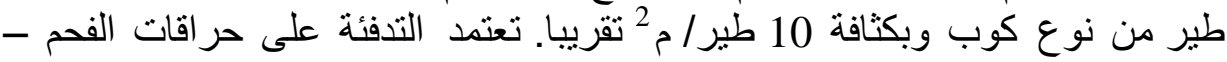

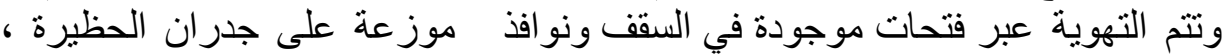

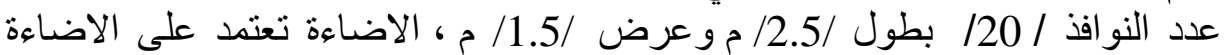

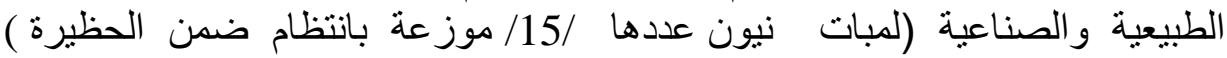

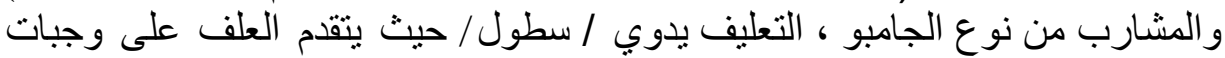
متعددة للفروج ، الفرشة نشارة خشب الب سماكتها 4 سم.

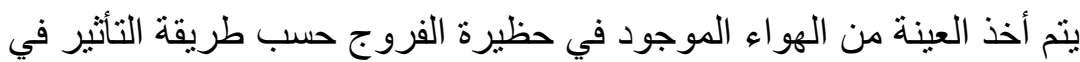
وسط سائل / The All-Glas Impinger 30 (

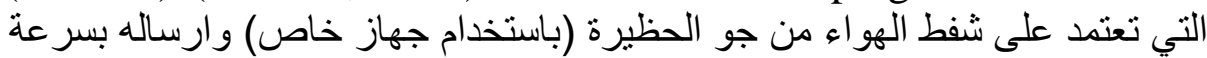

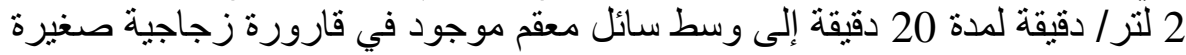

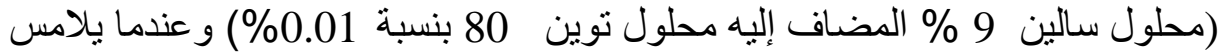

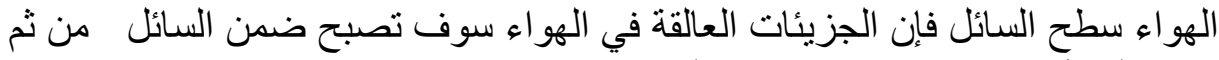

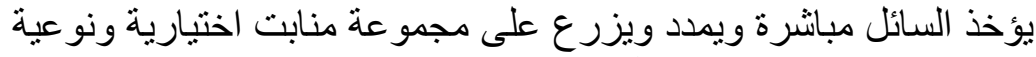
(Grabinska, 1999)

1- آجار مغذي Nutrient Agar: يستخدم من أجل تعداد الجراثيم أليفةالحرارة

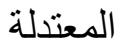

2 - منبت تثنابمان Chapman Media : يستخدم من أجل تعداد المكور ات العنقودية 3 - منبت ادوارد Edwards Media 4 - منبت مكونكي 3 منب Mackonkey Media: بستخدم من أجل تعداد الجراثيم سلبية

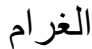
5 - منبت سابرود Sabouroud Dextrose Media: يستخدم من أجل تعداد الفطور 
يتم تحضين المنابت الجرثومية على 37 37 لمدة 24 25- 48 ساعة أما الفطور

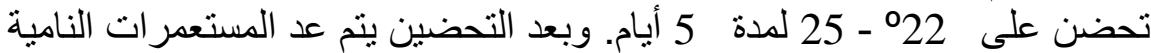

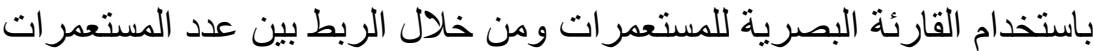

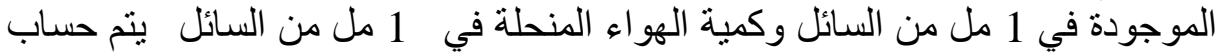

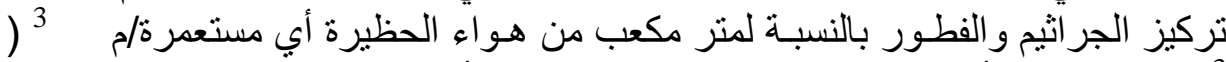

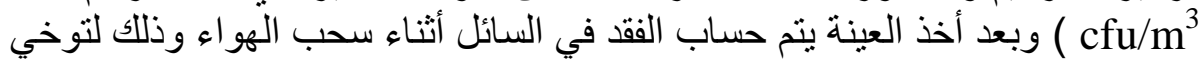

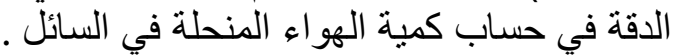

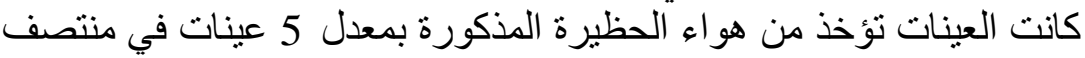

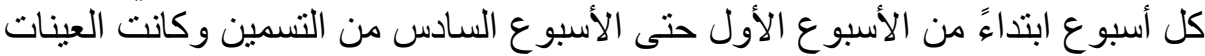

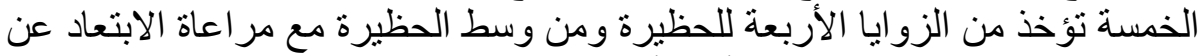

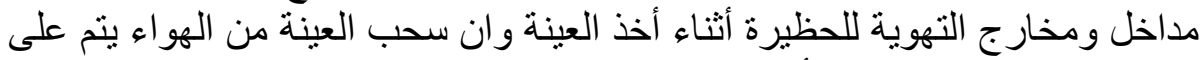

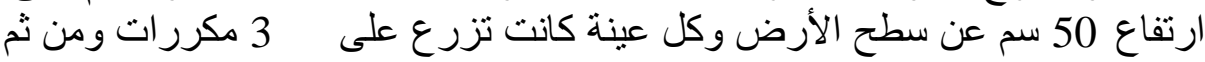

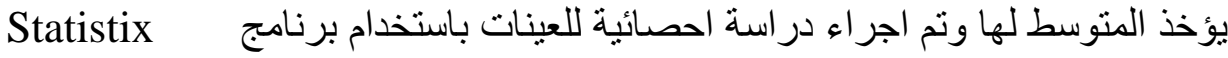
(1996): Analytical Softwar Version 1.0 , USA شملت الدر اسة المتوسط الحسابي ـ الانحر اف المعياري- القيمة الدنيا ــ القيمة

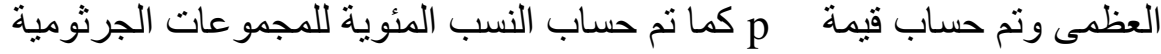

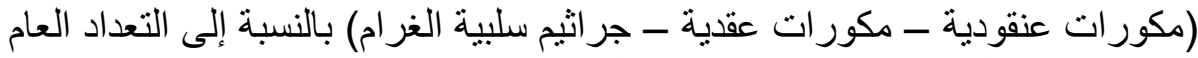

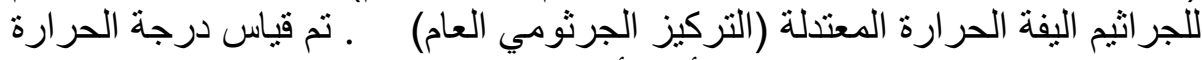
و الرطوبة والاضاءة وكثافة الطيور أثناء أخذ العينة.

\section{تحديد الهوية الجرثومية:}

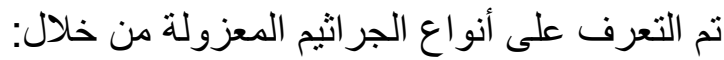

1 - شكل المستعمر ات النامية على الجئ المنابت التمبية التهيزية.

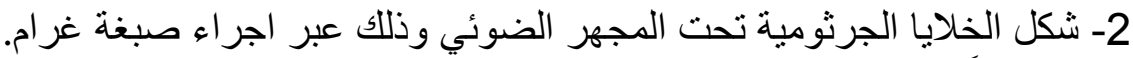

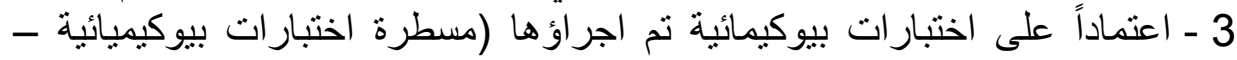

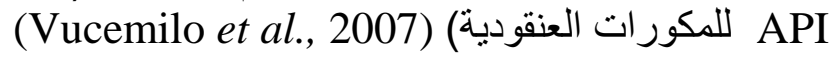

\section{RESULTS}

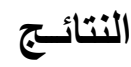

1 - الجراثيم أليفة الحرارة المعتدلة (التركيز الجرثومي العام):

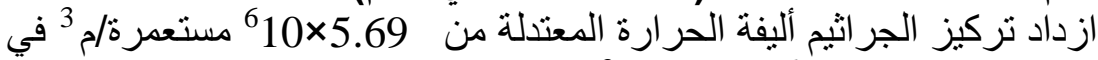

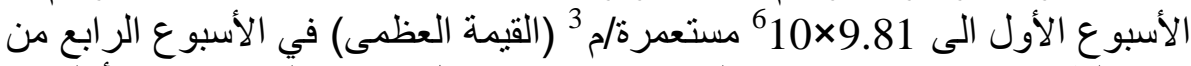

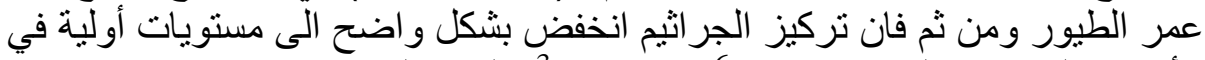

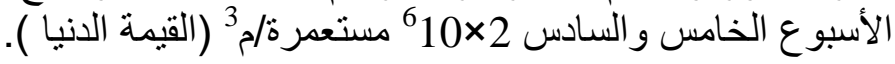




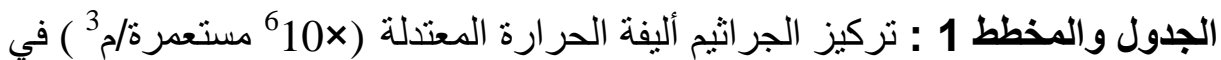

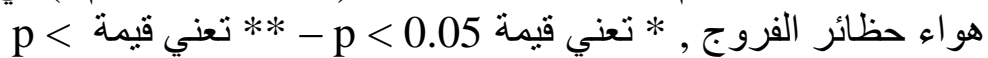

0.01

\begin{tabular}{|c|c|c|c|c|}
\hline العظمى القيمة & القيمة الدنيا & الالنحراف & الحسابي & العمر (الأسبوع) \\
\hline 9.12 & 2.77 & 2.70 & 5.69 & 1 \\
\hline 12.77 & 2.34 & 3.89 & 8.07 & 2 \\
\hline 15.11 & 5.03 & 4.68 & 8.70 & 3 \\
\hline 13.53 & 2.57 & 4.30 & 9.81 & 4 \\
\hline 7.97 & 1.96 & 2.63 & $* 4.07$ & 5 \\
\hline 3.01 & 1.65 & 0.57 & 2.00 & 6 \\
\hline
\end{tabular}

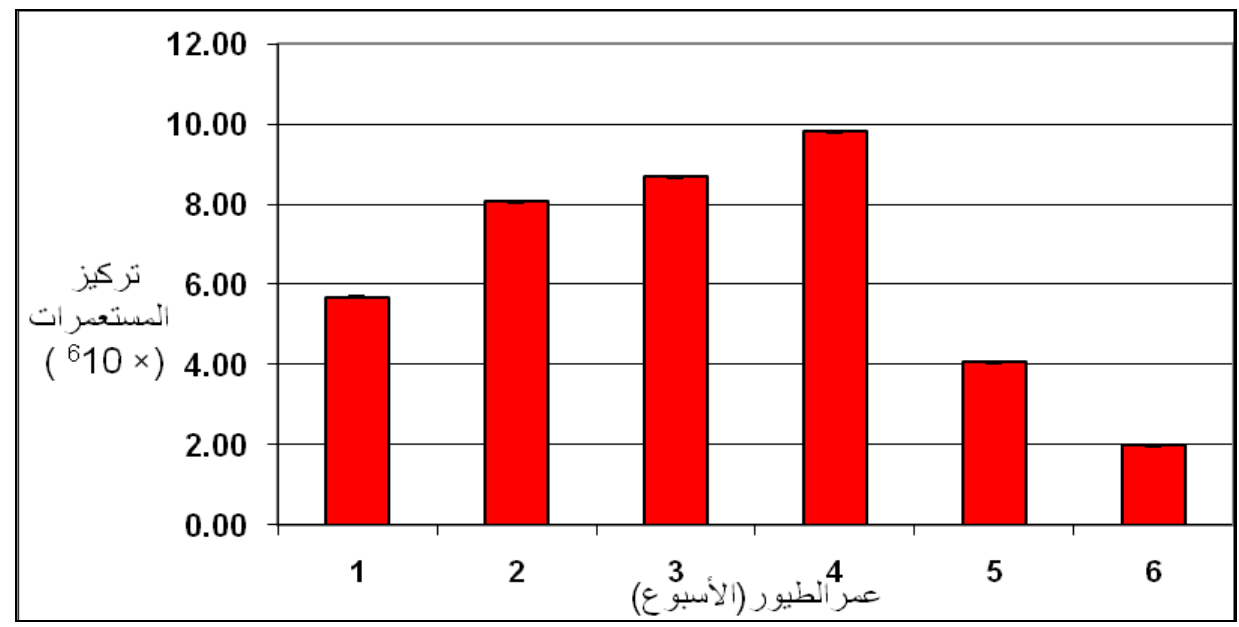

2 - المكورات العنقودية:

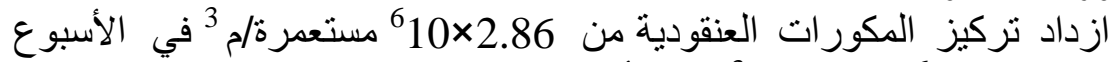

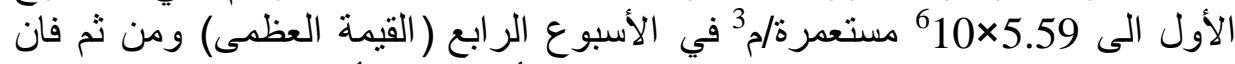

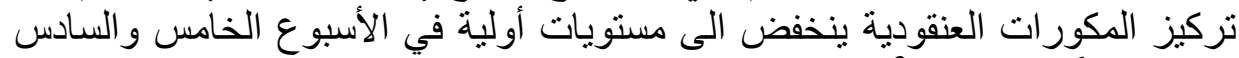
10×1.20 
الجدول والمخطط 2: تركيز المكورات العنقودية (x 106 مستعمرة/م ${ }^{6}$ 2 ) في هواء

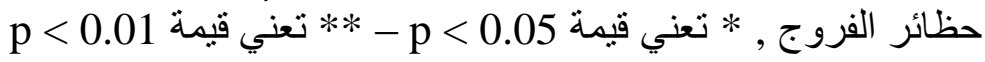

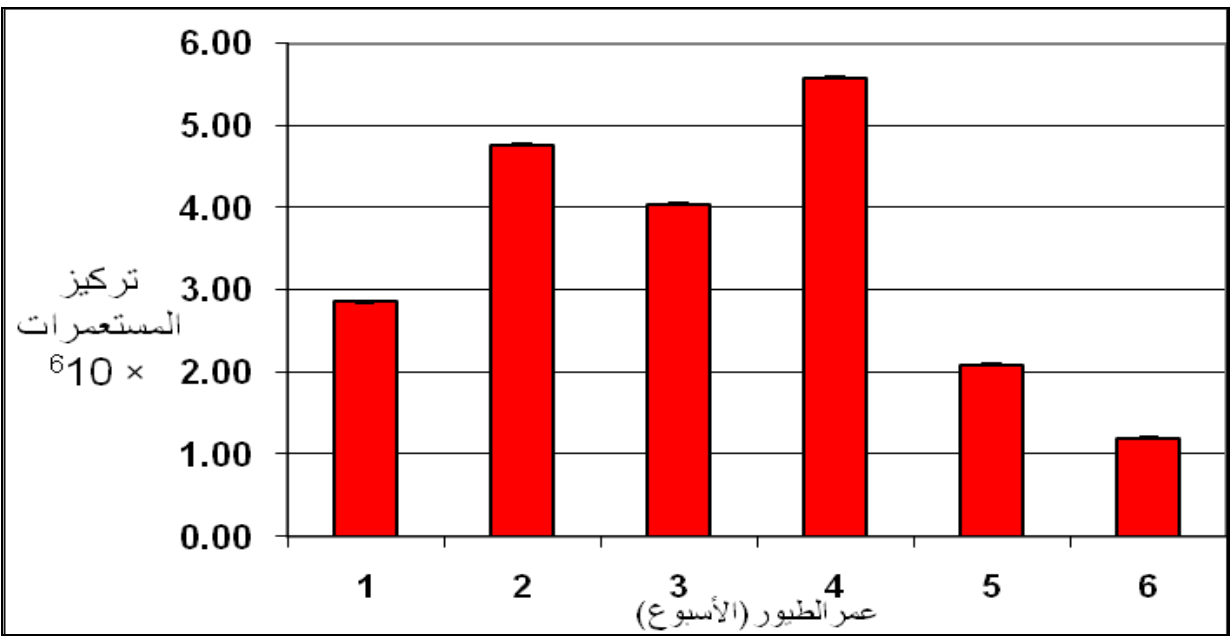

3 - 2 - المكورات العقدية:

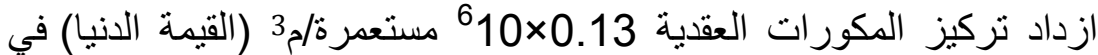
الأسبوع الأول الى 0.97×670 مستعمرة/33 في الأسبوع الر ابع ( القيمة العظمى)

\begin{tabular}{|c|c|c|c|c|}
\hline القيمةالعظمى & القيمة الدنيا & الانحر اف المعياري & الحستوسطي & $\begin{array}{c}\text { (الأسبوع) } \\
\text { (العمر }\end{array}$ \\
\hline 5.26 & 1.09 & 1.72 & 2.86 & 1 \\
\hline 6.52 & 0.78 & 2.30 & 4.77 & 2 \\
\hline 7.91 & 2.51 & 2.58 & 4.05 & 3 \\
\hline 7.35 & 3.68 & 1.72 & 5.59 & 4 \\
\hline 5.07 & 1.16 & 1.67 & $* 2.09$ & 5 \\
\hline 2.26 & 0.90 & 0.58 & 1.20 & 6 \\
\hline
\end{tabular}

و ون ثم فان تركيز المكورات العقدية بنخفض في الأسبوع الخامس والسادس. 
الجدول والمخطط 3: نركيز المكورات العقدية (x 106 مستعمرة/م 3 ) في هو اء حظائر

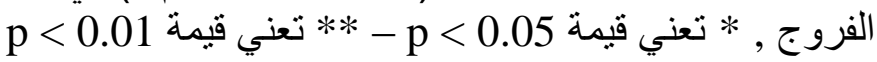

\begin{tabular}{|c|c|c|c|c|}
\hline القيمة العظمى & الدنيا & الالحعراف & الحسابي & العمر (الأسبوع) \\
\hline 0.15 & 0.08 & 0.03 & 0.13 & 1 \\
\hline 0.99 & 0.21 & 0.30 & $* 0.47$ & 2 \\
\hline 0.86 & 0.22 & 0.27 & 0.56 & 3 \\
\hline 1.25 & 0.66 & 0.26 & $* 0.97$ & 4 \\
\hline 0.51 & 0.07 & 0.19 & $* 0.28$ & 5 \\
\hline 1.13 & 0.38 & 0.31 & 0.57 & 6 \\
\hline
\end{tabular}

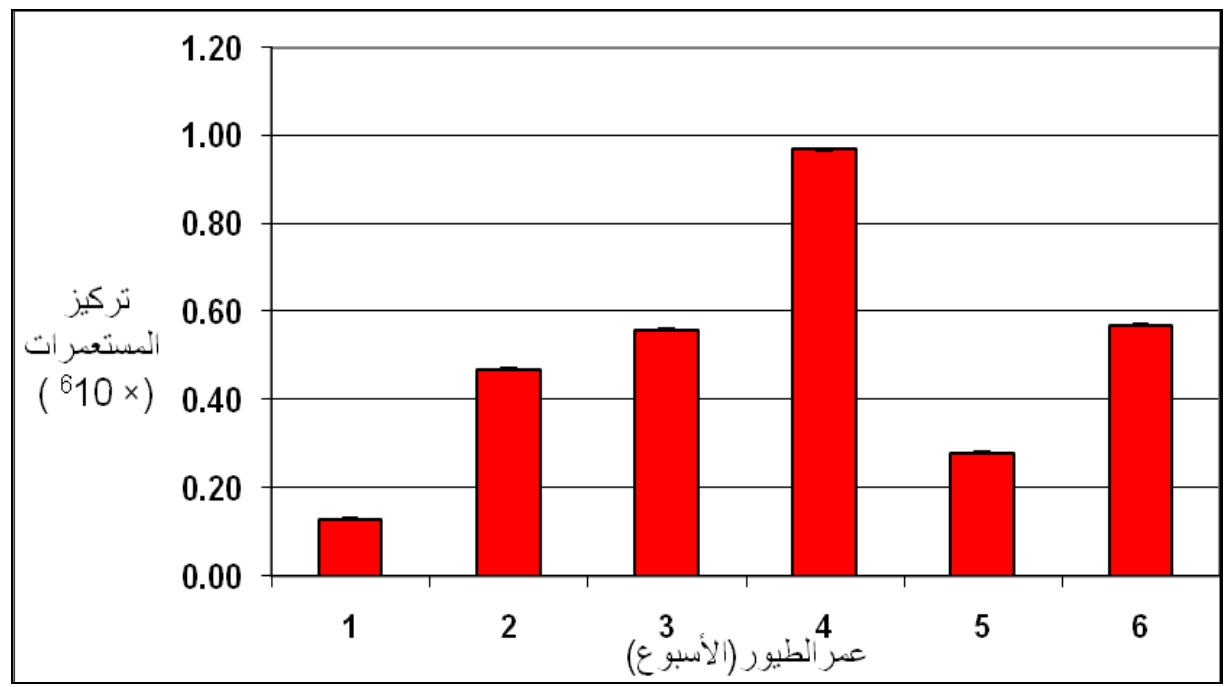

4 - الجراثيم سلبية غرام: 


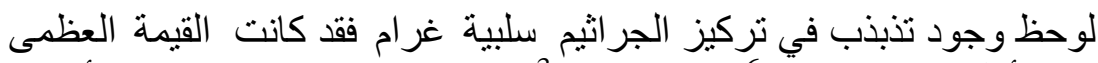

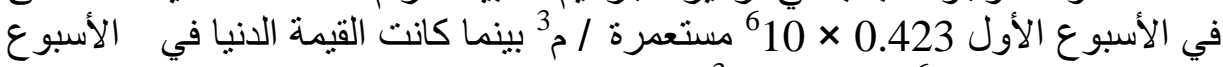

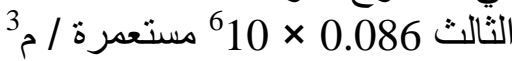

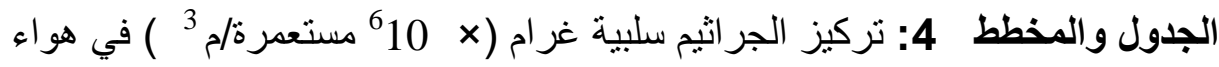

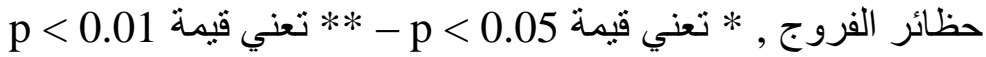

\begin{tabular}{|c|c|c|c|c|}
\hline القيمة العظمى & القيمة الدنيا & $\begin{array}{c}\text { الانحيارير افSD } \\
\text { SD }\end{array}$ & الحسابي & العمر (الأسبوع) \\
\hline 0.729 & 0.219 & 0.279 & 0.423 & 1 \\
\hline 0.284 & 0.141 & 0.050 & 0.213 & 2 \\
\hline 0.144 & 0.072 & 0.032 & $* 0.086$ & 3 \\
\hline 0.515 & 0.147 & 0.169 & $* 0.265$ & 4 \\
\hline 0.217 & 0.072 & 0.060 & 0.131 & 5 \\
\hline 0.300 & 0.075 & 0.098 & 0.135 & 6 \\
\hline
\end{tabular}

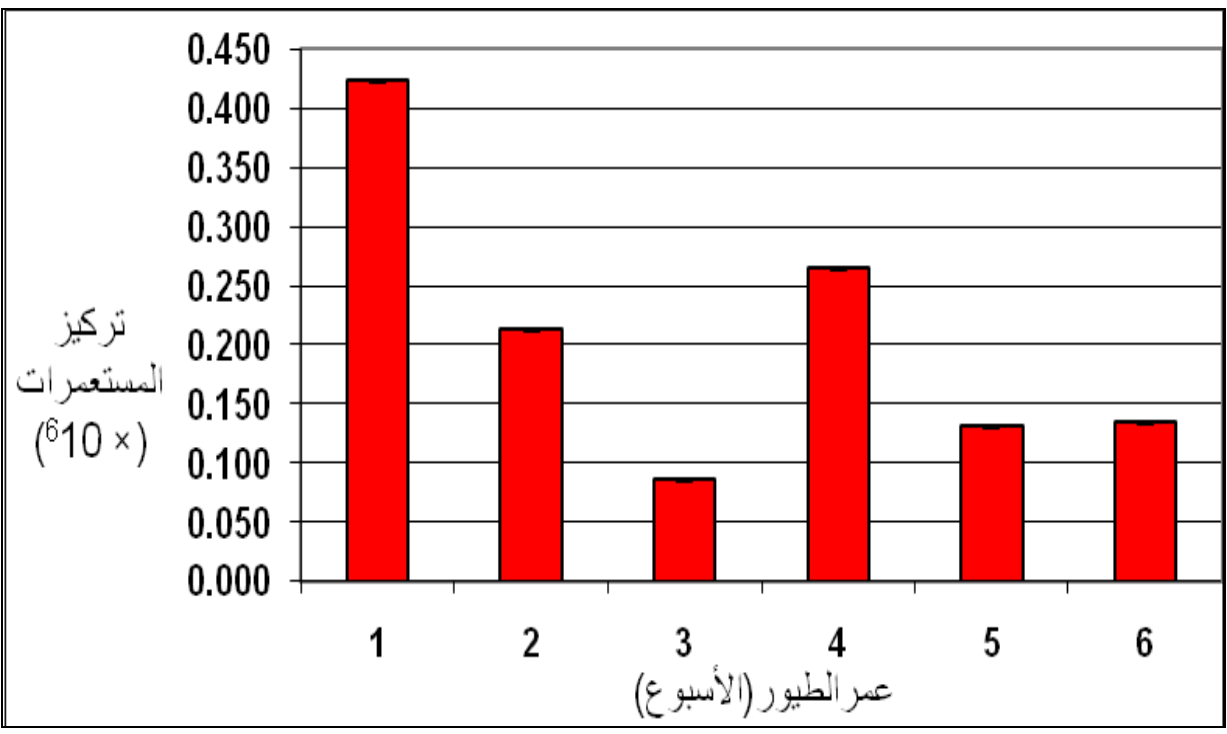




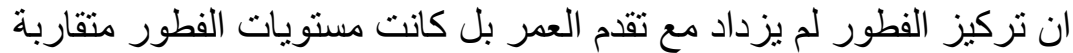

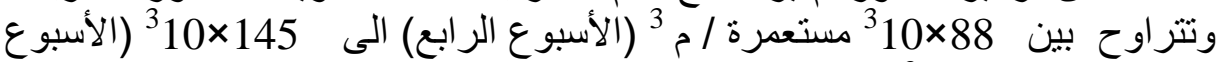

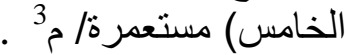

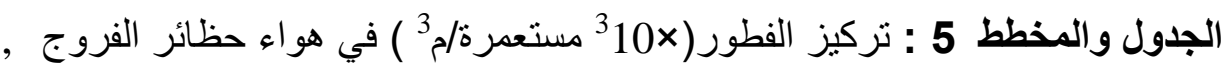

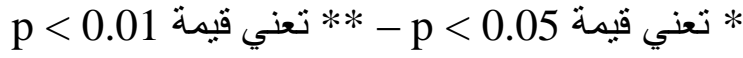

\begin{tabular}{|c|c|c|c|c|}
\hline العظمى & القيمة الدنيا & الانحياري افSD المي & الحسابي & العمر(الأسبوع) \\
\hline 292 & 75 & 90 & 132 & 1 \\
\hline 284 & 71 & 86 & 142 & 2 \\
\hline 216 & 72 & 60 & 129 & 3 \\
\hline 147 & 74 & 32 & 88 & 4 \\
\hline 289 & 73 & 88 & 145 & 5 \\
\hline 256 & 76 & 67 & 105 & 6 \\
\hline
\end{tabular}

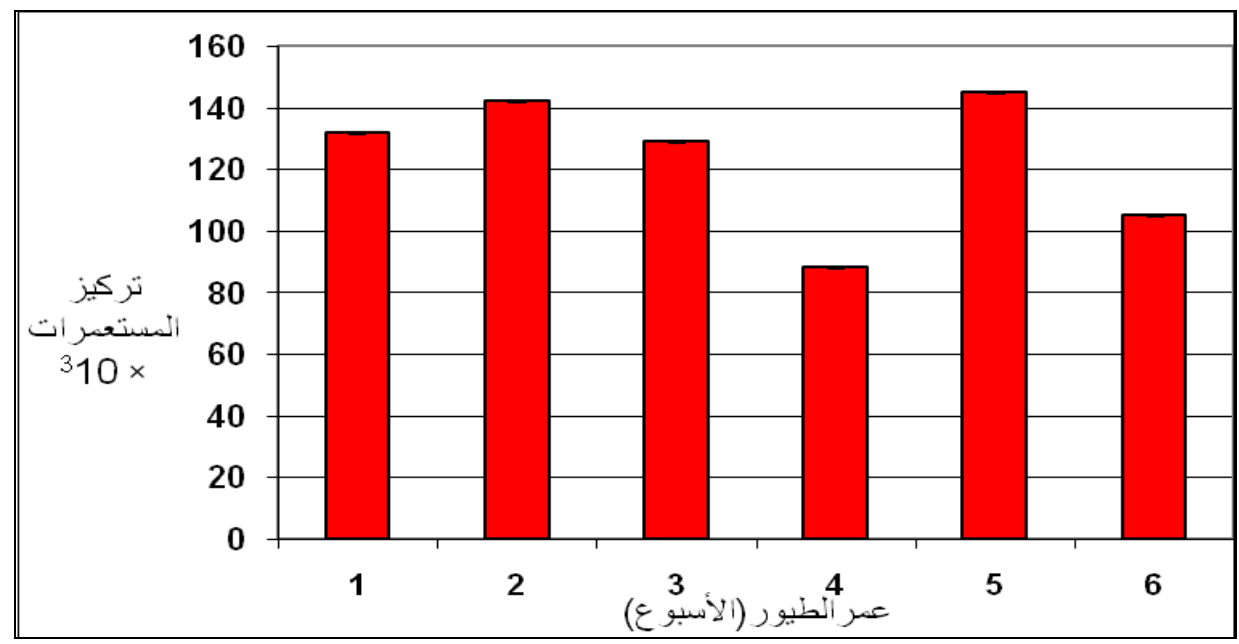

النسب المئوية للمجموعات الجرثومية من التركيز الجرثومي العام (تركيز الجراثيم

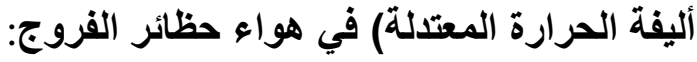


ان نسبة المكورات العنقودية كانت تمثل 26.6-60\% من من التركيز الجرثومي

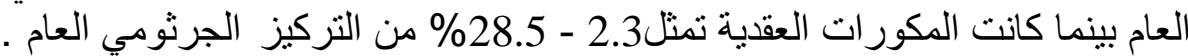

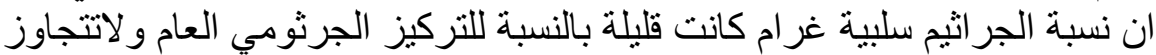
\% $7.4-1$

الجدول والمخطط 6: النسب المئوية للمجموعات الجرثومية من التركيز الجرثومي العام في هو اء حظائر الفروج.

\begin{tabular}{|c|c|c|c|}
\hline الجراثيم سلبية & المكور ات العقدية & المكورات العنقودية & (الأسبوع ) العمر \\
\hline $7.4 \%$ & $2.3 \%$ & $50.3 \%$ & 1 \\
\hline $2.6 \%$ & $5.8 \%$ & $59.1 \%$ & 2 \\
\hline $1 \%$ & $6.4 \%$ & $46.6 \%$ & 3 \\
\hline $2.7 \%$ & $9.9 \%$ & $57 \%$ & 4 \\
\hline $3.2 \%$ & $6.9 \%$ & $51.4 \%$ & 5 \\
\hline $6.8 \%$ & $28.5 \%$ & $60 \%$ & 6 \\
\hline
\end{tabular}

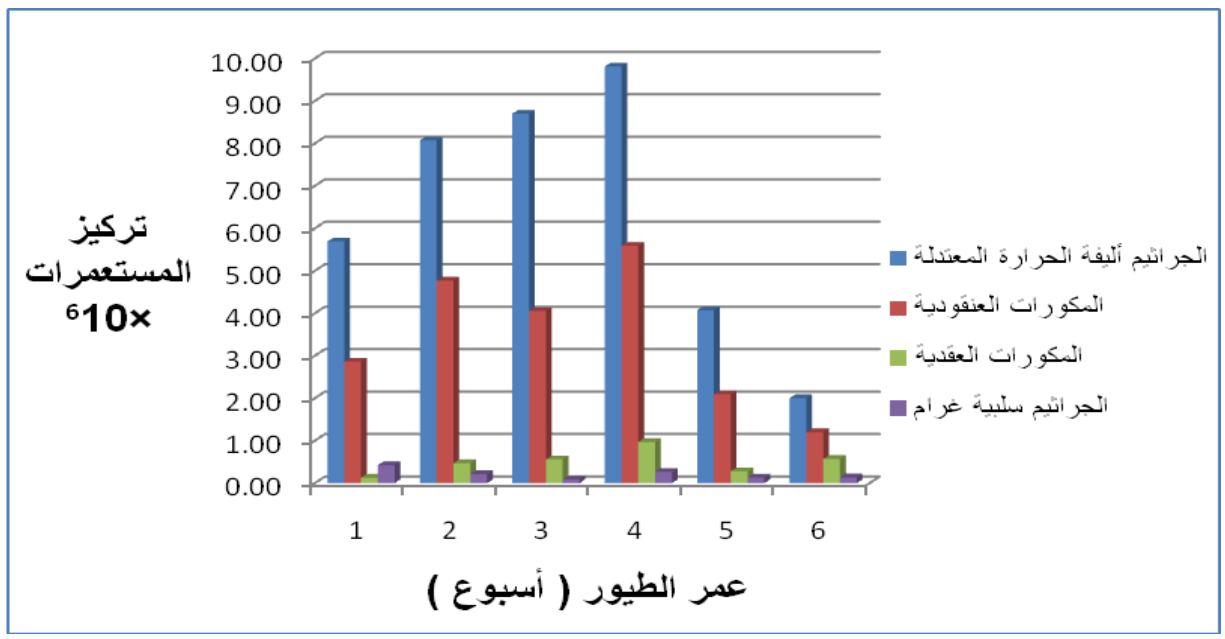

الجدول 7: نتائج قياس درجات الحرارة والاضاءة والرطوبة وكثافة الطيور عند أخذ العينات 
Assiut Vet. Med. J. Vol. 57 No. 130 July 2011

\begin{tabular}{|c|c|c|c|c|}
\hline الكثافة (العدد/ م²) & الاضـاءة (لكس) & الرطوبة (\%) & الحر ارة & العمر (الأسبو ع) \\
\hline 32.5 & 45 & 48.5 & 27.3 & 1 \\
\hline 26 & 50 & 71.5 & 22.5 & 2 \\
\hline 18 & 40 & 72.5 & 21.4 & 3 \\
\hline 15.5 & 52.6 & 75 & 21.1 & 4 \\
\hline 12.4 & 63.8 & 80.3 & 20.3 & 5 \\
\hline 12.2 & 75.5 & 85 & 19.5 & 6 \\
\hline
\end{tabular}

\section{DISCUSSION}

المناقشــة

ان تركيز الجراثيم أليفة الحرارة المعتللة (النتكيز الجرثومي العام ) في هواء

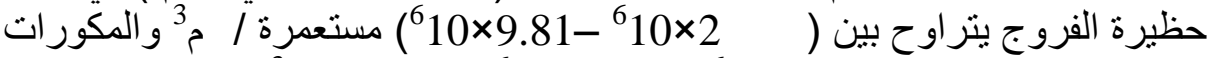

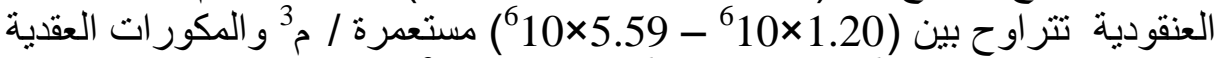

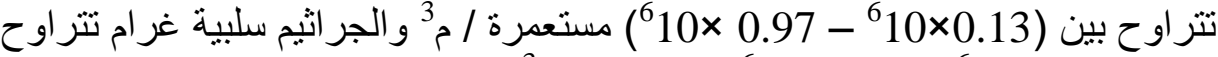

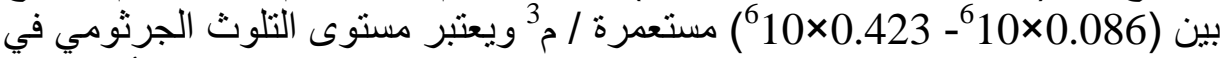

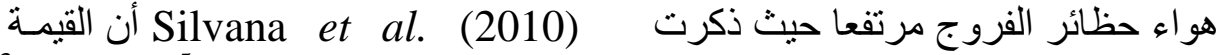

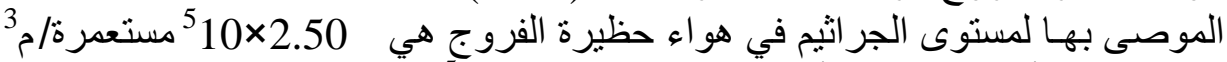

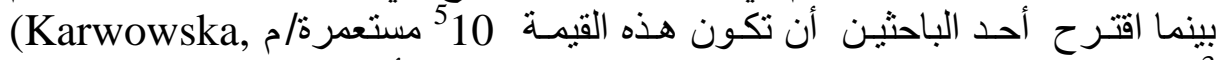

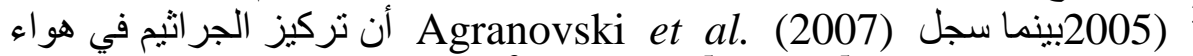

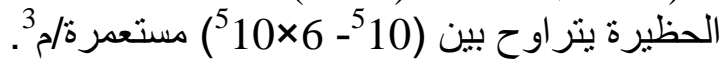

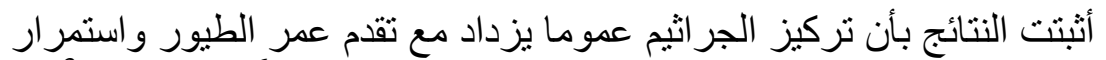

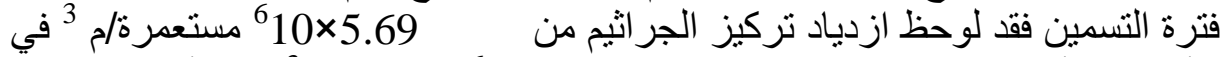

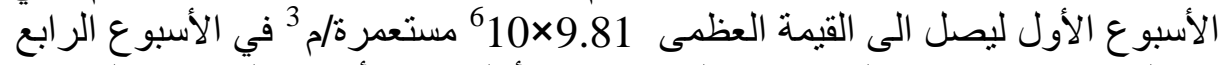

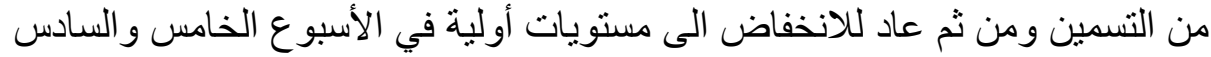

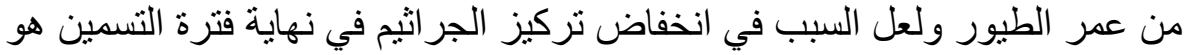

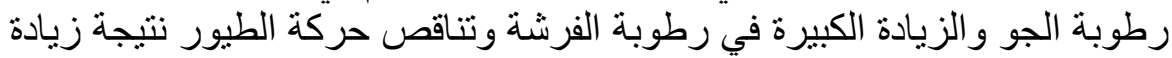

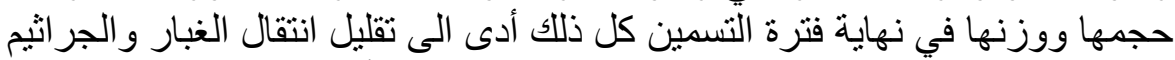

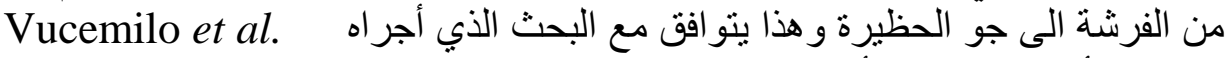

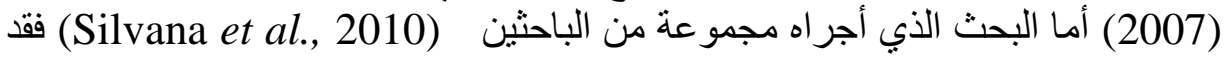

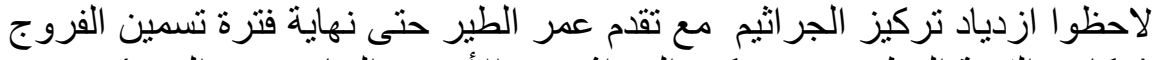

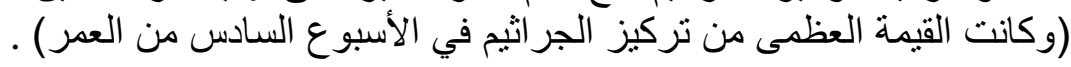


ان المكور ات العنقودية كانت تمثل (46.6-60) \% من من الجر اثيم اليفة الحرارة

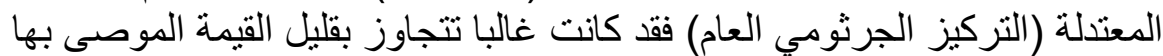

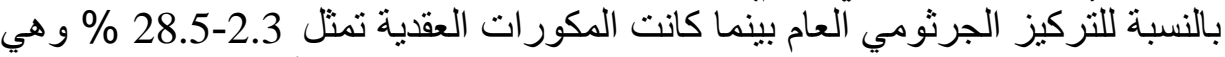

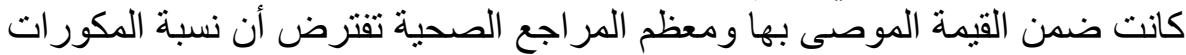

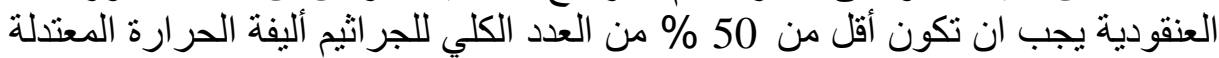

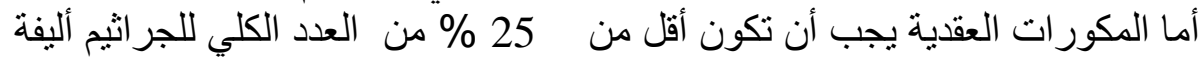

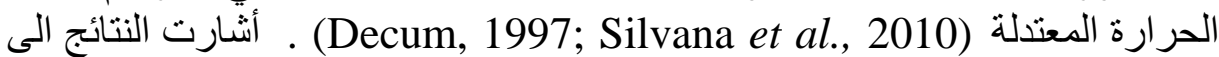

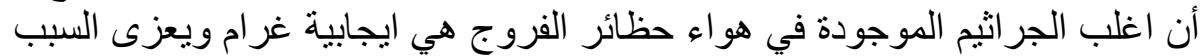

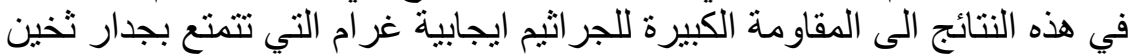

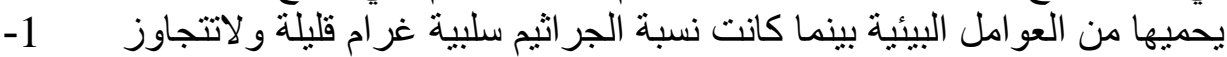

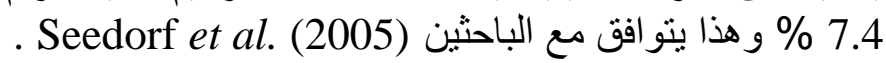

كانت أعداد الفطور غير مرتفعة وضمن الحدود المقبولة في حظيرة الفروج

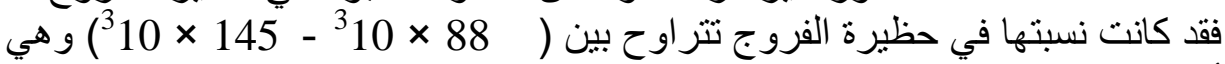

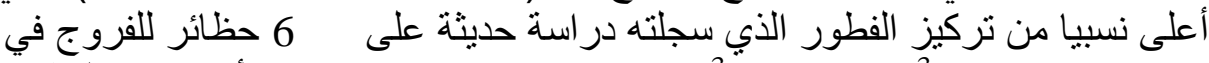

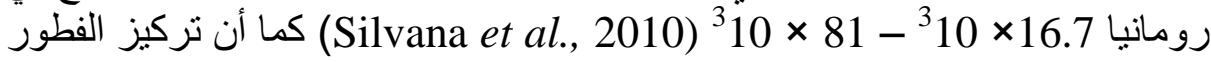

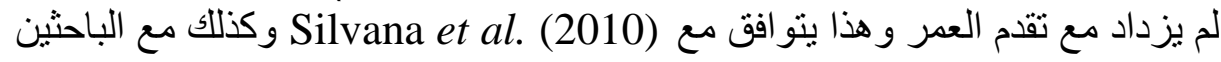
. Vucemilo et al. (2007)

\section{REFERENCES}

Afanasyva, G. (1997): Opriedielenyje bakterialnich endotoksins kak alternativnij metod. Baltic J. Lab. Animal Sci. 7: 187-199.

Agranovski, V.; Reponen, T. and Ristovski, ZD. (2007): Survey of Bioaerosol Emissions from Australian Poultry Buildings. European Aerosol Conference, Salzburg: Abstract T04A003.

Bakutis, B.; Monstviliene, G. and Januskeviciene (2004): Analyses of Airborne Contamination with Bacteria, Endotoxins and Dust in Livestock Barns and Poultry Houses. Acta Vet. Brno, 73: 283-289.

Decun, M. (1997): Airborne microflora, In: Veterinary Hygiene and the Protection of the Environment, Helicon, Timisoara, Romania, pp. 82-85.

Eduard, W. (1997): Exposure to Non- Infectious Microorganisms and Endotoxins in Agriculture. Annals of Agricultural and Environmental Medicine, 4, 2. 
Golbabaei, F. and Islami, F. (2000): Evaluation of Workers' Exposure to Dust, Ammonia and Endotoxin in Poultry Industries at the Province of Isfahan, Iran. Industrial Health, 38: 41- 46.

GrabInska-Oniewskaa, (Ed.) (1999): Laboratory exercises on microbiology (In Polish). Oficyna Wydawnicza PW, Warszawa.

Harry, E.G. (1978): 'Air pollution in farm buildings and methods of control: A review', Avian Pathology, 7, 4: 441-454.

Hartung, J. (1994): Environment and Animal Health In: Livestock Housing Book, Edited by C.M. Wathes und D.R. Charles, 25-49.

Hartung, J. and Schulz, J. (2005): Risks caused by bio-aerosols in poultry houses University of Veterinary Medicine Hannover, Bünteweg 17p, 30559 Hannover, Germany. E-mail: itt@ tihohannover.de.

Karwowska, E. (2005): Microbiological air contamination in farming environment. Polish Journal of Environmental Studies 14: 445-449.

Müller, W. (1987): Origin, quantity and quality of microbial emissions in animal houses. In: Strauch D. (ed.): Animal Production and Environmental Health. Amsterdam, Elsevier Science Publishers, B6: 66-71.

Muller, W. and Wieser, P. (1987): Dust and microbial emissions from animal production. Strauch D (Hrsg.). Animal production and environmental health. Elsevier, Amsterdam-Oxford- New YorkTokio, pp. 47-89.

Nowak, D. (1998): Die Wirkung von Stalluftbestandteilen, insbesondere in Schweineställen, aus arbeitsmedizinischer Sicht. Dtsch. tierärztl. Wschr., 105: 225-234.

Radon, K.; Danuser, B.; Iversen, M.; Monso, E.; Weber, C.; Hartung, J.; Donham, K.J.; Palmgren, U. and Nowak, D. (2002): Air contaminants in different European farming environments. Ann. Agric. Environ. Med., 9: 41-48.

Schulz, J.; Seedorf, J. and Hartung, J. (2005): Estimation of a "safe distance" between a natural ventilated broiler house and a residential dwelling. In A. Krynski \& R., Wrzesien, eds. Proceedings, XIIth International Congress on Animal Hygiene of the ISAH, Volume 2 Animals and Environment, held Warsaw, Poland, 4-8 September 2005. International Society for Animal Hygiene. 
Seedorf, J. (2005): Messung luftgetragener biologischen Komponenten (Measurement of airborne biological components). In: Luftgetragene biologische Belastungen und Infektionen am Arbeitsplatz Stall. Kuratorium für Technik und Bauwesen in der Landwirtschaft (Association for Technology and Structures in Agriculture, KTBL), Darmstadt, KTBL-Schrift 436: 66-71.

Seedorf, J.; Schröder, M.; Linkert, K.H. and Wathes, C.M. (1998): Concentrations and emissions of airborne dust in livestock buildings in Northern Europe. Journal of Agricultural Engineering Research, 70(1): 59-77.

Seedorf, J. and Hartung, J. (2002): Stäube und Mikroorganismen in der Tierhaltung. KTBL-Schrift 393, Landwirtschaftsverlag GmbH, Münster, 166 Seiten.

Seedorf, J.; Schulz, J. and Hartung, J. (2005): Outdoor measurements of airborne emission of staphylococci from a broiler barn and its predictability by dispersion models. WIT Transactions on Ecology and the Environment, 85: 33-42.

Silvana, P.; Cristin, B.; Cristina, H.; Razvan, S. and Evandrea, L. (2010): The Microbiologic Quality of the Air in Broiler Houses Scientific Papers: Animal Science and Biotechnologies, 43 (2).

Vučemilo, M.; Vinković, B.; Tofant, A.; Šimpraga, B.; Pavičić, Ž. and Matković, K. (2005): Microbiological air contamination in intensive poultry breeding. In: Proc. $12^{\text {th }}$ Int. Congress ISAH, September 4-8, Warsaw, Poland, 1: 127-129.

Vučemilo, MK.; Matković1, B.; Vinković, S.; Jakšić, K. and Granić, N. (2007): The effect of animal age on air pollutant concentration in a broiler house. Czech J. Anim. Sci., 52, (6): 170-174. 
Assiut Vet. Med. J. Vol. 57 No. 130 July 2011 